\title{
Mobility Enhanced Smart Antenna Adaptive Sectoring for Uplink Capacity Maximization in CDMA Cellular Network
}

\author{
Alex Wang and Vikram Krishnamurthy
}

\begin{abstract}
In this paper, adaptive sectoring of a CDMA cellular network is investigated, and the aim is to maximize the uplink capacity by utilizing mobiles' spatial information. One important feature of the algorithm developed is that it does not depend on tracking individual mobile, but rather on the statistics of mobiles. The distribution of mobiles is modeled as a spatial Poisson process, whose rate function quantizes mobile concentration and is inferred with a Bayesian estimator based on the statistics of network traffic. In addition, the time dynamics of the rate function is assumed to evolve according to mobiles' mobility pattern and it is formulated using the Influence model. With the knowledge of mobiles' spatial distribution, the interference and thus the outage probability of different sector partitions of a cell can be computed. The adaptive sectoring problem is formulated as a shortest path problem, where each path corresponds to a particular sector partition, and the partition is weighted by its outage probability. In simulation examples, a hot spot scenario is simulated with the adaptive sectoring mechanism, and it is observed that load balancing between sectors is achieved and which greatly reduces the effect of hot spot.
\end{abstract}

Index Terms-Mobility estimation, adaptive sectoring, smart antenna, and CDMA uplink capacity.

\section{INTRODUCTION}

$\mathbf{I}$ $\mathrm{N}$ wireless cellular networks, CDMA is a promising technology to offer high quality and robust voice/data services. Its RAKE receiver design and soft handoff greatly increase the robustness against multipath and fast fading environment. However, the ever increasing demand for the wireless services is continuously challenging the capacity limit of CDMA networks. In this paper, the application of adaptive sectorization to increase the network capacity is studied.

It is well known that CDMA systems are interference limited, and sectoring has been an effective mean of increasing the network capacity by introducing spatial domain orthogonalization to the system. The conventional method applied in, for example, GSM and IS-95 employs $120^{\circ}$ or $60^{\circ}$ sectoring to achieve better reuse of network resources. However, one major drawback of this scheme is its inflexibility in dealing with non-stationary and non-uniform mobile distribution. For example, hot spots can cause outage in a sector while other sectors have light traffic.

Paper approved by X. Wang, the Editor for Multiuser Detection and Equalization of the IEEE Communications Society. Manuscript received February 23, 2006; revised January 10, 2007.

The authors are with the University of British Columbia, Department of Electrical and Computer Engineering, 2112 Acadia Road, Vancouver, British Columbia V6T 1R5, Canada (e-mail: alexw@ece.ubc.ca).

Digital Object Identifier 10.1109/TCOMM.2008.060117.
In this paper, we extend the conventional sectorization by allowing base stations to observe network traffic and adaptively sectorize cells accordingly. The dynamic sectorization is achieved by deploying smart antenna systems at base stations. While smart antenna is often associated with adaptive beam forming, our approach is fundamentally different. Even though both approaches utilize the spatial domain, while beam forming directs dedicated beam to each mobile, sectorization spans cells with few main beams with each beam corresponds to a sector. Smart antenna system is supported in many wireless standards; [1] describes auxiliary pilot support with switched beam in CDMA2000, [2] details the application of smart antenna in IS-95 and [3] describes how dedicated pilot symbols in WCDMA systems can render future deployment of smart antenna easier.

Many researchers, [4]-[6], have investigated the adaptive sectoring problem. [4] considers the case with fixed and known mobile locations, as in the wireless local loop, and formulates the adaptive sectoring as a shortest path problem. The problem is solved for two cases: minimizing the mobiles' total transmit power and minimizing the base station's total received power. Our approach follows the modeling technique used in [4] and extends it to take mobile movement into account. [6] assumes a spatial Poisson process with known intensity function $\lambda$, and the probability of having $k$ mobiles in an area $A$ is given by the Poisson distribution $P(k, A)=\frac{(\lambda A)^{k}}{k !} e^{-\lambda A}$. By fixing $k$ and $P=\sum_{j=k}^{\infty} P(j, A)$, and replace $A$ by $\frac{r^{2} \theta}{2}$ where $r$ is cell radius and $\theta$ is a sector's angle span, $P$ is the probability of having more than $k$ users in $\theta$. Adaptive sectoring is computed by an iterative method which reduces $\theta$ when $k$ is above a certain threshold. [5] continuously monitors SINR (signal to interference and noise ratio) of all the users, and sectorize cells to equalize SINR in all sectors. However, in each of the above solutions, there are certain limitations. While the work in [4] is designed for wireless local loop, it does not work with constantly moving mobiles. In [6], the success of the algorithm depends on the knowledge of mobile concentration. Moreover, the SINR-based sectoring in [5] may be unstable because of the shadowing and fast fading in the measurement of SINR [7].

The major difference between adaptive and conventional sectoring is the system's responsiveness to changes in mobile distribution. Movement of people is observed to follow certain patterns [8], and in this paper, a mobility-enhanced traffic model is developed to capture the dynamic of mobile 
concentration over period of a day from the network traffic observed. Based on the estimated mobile distribution, the sectoring problem is solved to maximize the uplink capacity.

The main contributions of the paper:

1) A mobility-enhanced traffic model is developed. Mobile distribution is modeled as a spatial Poisson process with time-varying rate function. The rate functions of different locations are assumed to evolve according to the mobiles' aggregate mobility pattern and which is formulated with the Influence model [9]. If the dynamics is formulated in ordinary Markov chain, the curse of dimensionality greatly limits the applicability of the model.

2) Recursive MAP estimator of the spatial Poisson's rate function given the network traffic. It provides real time tracking of mobile distribution over the network. $R e$ mark: Global MAP estimator which tracks the joint a posteriori distribution of the entire network's rate functions is too computationally intensive for real time applications. The MAP estimator developed tracks the marginal a posteriori distribution of each individual location's rate function.

3) Formulation of the adaptive sectoring problem as a shortest path problem for changing mobile distribution. The modeling technique was first applied in [4] to sectorize cells in wireless local loop based on individual mobiles, and it is extended to work with the aggregate mobility pattern in this paper.

The paper is organized as follows. Sec. II defines the adaptive sectoring problem and formulates the related models. Sec. III develops the algorithms for solving the adaptive sectoring problem. Sec. IV presents the simulation results and Sec. V concludes the paper.

\section{Model Definitions AND Formulation OF AdAPTIVE SECTORING PROBLEM}

In this section, adaptive sectoring of CDMA networks is formulated as a sequence of uplink capacity maximization problems, and the adaptive capability deals particularly with the mobility pattern of mobile users. Uplink capacity is chosen as the cost function because it is the limiting factor [10], [11], and it is measured by the probability of interference at a base station exceeding a certain threshold value [10], [12], i.e., the outage probability. In summary, the aim is to formulate a outage probability minimization problem for CDMA uplinks with adaptive sectorization.

In order to compute outage probability at each base station, knowledge of mobiles' whereabouts is necessary. Yet, as the number of mobiles increases, tracking individuals is computationally intensive, and it may lead to frequent sectoring because of their various movement patterns. The approach taken considers mobiles' aggregate movement, and it is implemented by dividing the network into areal units and tracking the time evolution of mobile concentration at each unit. The tracked mobile concentration in turn enables the computation of outage probability. The adaptive sectoring problem consists of three components: the formulation of the sectoring problem as an optimization problem, the mobility model for the mobile users, and the numerical computation of outage probability. The components are established in this section.

\section{A. Formulation of Adaptive Sectoring Problem}

The network model consists of hexagonal cellular networks and, as illustrated in Fig. 1, each cell is divided into six equally spaced areal units called subareas. As will be described in further detail in the next two subsections, mobility of the mobiles is modeled as a graph, where each node represents the mobile concentration in each subarea and the nodes are connected by edges indicating the prior assumption of the movement patterns of the mobiles.

In this subsection, given the mobile concentration, discrete sectoring is considered and formulated as an optimization problem. Sectors at each base station are defined by the antenna's sector-beams, whose beamwidth is multiples of a subarea's angular span. Perfect beam pattern (no overlap between beams) is assumed, and thus mutual interference is ignored. Note the dimension of subareas defines the granularity of the model, i.e., finer tracking of mobile distribution is enabled by smaller subareas, but with higher computational load on the system.

A natural mathematical representation of the adaptive sectoring problem is with graph partitioning. The key advantage is that, under certain conditions, the partitioning problem has a one-to-one correspondence to a shortest path problem, and which is readily solvable. The modeling technique described was first applied in [4] to sectorize wireless local loops with stationary mobiles, and it is extended in this paper to deal with aggregate statistics of mobile movement. Because the rest of the paper builds on top of the model in [4], it is briefly summarized in this subsection.

Fig. 1 illustrates the graph theoretical representation of the cellular network. A cell is modeled as a ring of nodes where each node represents a subarea, and the sectoring problem is equivalent to the partitioning of nodes (subareas) into subsets (sectors). Denote $A=\left\{a_{1}, a_{2}, \ldots, a_{M}\right\}$ as the nodes of the ring, and $\pi=\left\{S_{1}, \ldots, S_{N}\right\}$ the partition of $A$ into $N$ subsets, the partitioning is considered optimal if it minimizes the cost function $C(\pi)$, where $C(\pi)$ is the outage probability experienced in all sectors $\pi$. Denote the outage probability in each sector $S_{i}$ as $W\left(S_{i}\right)$, the adaptive sectoring problem is reduced to a graph partitioning problem with the following cost function:

$$
C(\pi)=\sum_{i=1}^{N} W\left(S_{i}\right)
$$

In general, the problem of optimally partitioning an arbitrary graph with an arbitrary cost function is a NP-hard optimization problem. However, it has been shown that the partitioning problem can be solved in polynomial time if the graph is a string and the cost function is separable.

Definition A function of $M$ variables, $f\left(x_{1}, x_{2}, \ldots, x_{M}\right)$, is separable if it can be expressed as a sum of $M$ functions of a single variable; i.e., $f\left(x_{1}, x_{2}, \ldots, x_{M}\right)=\sum_{i=1}^{M} f_{i}\left(x_{i}\right)$.

Theorem 1 If the cost function is separable, the problem of optimally partitioning a string can be reduced to a shortest 


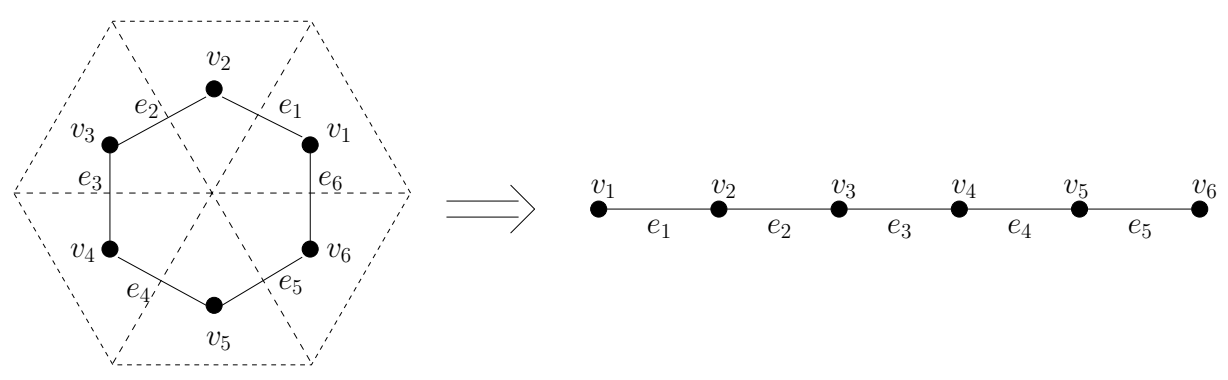

Fig. 1. The network is modeled as a hexagonal cellular network, and each cell is divided into six equally spaced areas called subarea. The Graph-theoretic representation of a cell is illustrated. The graph on the left is the ring representation of a cell, where each node is a subarea. (Sectors are disjoint subsets of nodes.) The graph on the right is one of six reduced string representations, where the edge $e_{6}$ is arbitrarily chosen and removed.

path problem. ( [4] applied this result to sectorize wireless local loop.)

Proof The proof can be found in [13].

The important observation of a ring is that it can be broken into a string if an edge is removed. In addition, with perfect sector-beam assumption, the computation of a sector's outage probability is independent of other sectors. In other words, the hypothesis of the above theorem holds. Fig. 1 illustrates the string representation where the edge $e_{6}$ is arbitrarily chosen and removed. It should be noted that the removal of an edge traded problem complexity with computational complexity since six strings are generated from one ring.

The mapping of the graph partitioning problem to a shortest path problem is illustrated by the construction of an acyclic network. However, the detail is omitted and it can be found in [4], [13]. The important point to note is that once the acyclic network is constructed, the weight at each edge corresponds to the outage probability in each sector, and it is changing in time. According to the above formulation, the adaptive sectoring problem can be viewed as a shortest path problem with a changing weight matrix, where the weight depends on the evolving statistics of mobile distribution. In Sec. II-B, a model is developed to track the statistics in each subarea, and Sec. III-C computes the outage probability.

\section{B. Mobility-Enhanced Traffic Model}

In this subsection, the aim is to develop a mobility model for mobile users that enables the tracking of mobile distribution by collecting its statistics. The first major problem that has to be addressed is the observability of mobility. In general, mobility is not observable, and it can only be indirectly observed through the network traffic processed at the base station. It is realized that if mobile users place calls according to a Poisson process, something could be said regarding the mobiles' spatial distribution according to the following theorem.

Theorem 2 Let $\Pi$ be a Poisson process of arriving calls at a base station with constant rate, $X$, from mobiles in an arbitrary subarea. Once the mobiles placed the call, they move at random around the subarea with independent trajectories. Let $E$ be a spatial subset of the subarea such that the probability of the mobile who called at time $s$ being in $E$ at a subsequent time $t$ is $p(s, t)$. Then the number of mobiles in $E$ at time $t$ has a Poisson distribution with mean

$$
u(t)=\int_{0}^{t} X p(s, t) d s .
$$

Assuming uniform distribution for $p(s, t)$ over the subarea, the distribution of the mobiles in the subarea is a spatial Poisson process with rate equals to that of the arriving calls.

Proof The proof can be found in [14], (pg 49 Bartlett's Theorem).

In Theorem 2, a number of assumptions are made, and it is worthwhile going into the details.

Assumption 1 The arrival process at the base station is a Poisson process with constant rate.

The arrival process referred to in the Theorem is the connection requests made by mobiles in the subarea. For example, the number of times Access Channel is requested in IS95 or CDMA2000. From the study of broadband network traffic [15], the connection request is generally modeled as an inhomogeneous Poisson process. The additional assumption is that the rate function, $X$, in a subarea is a jump process with finite states, and jumps occur on a hourly basis.

Assumption 2 Mobiles are distributed uniformly over the subarea.

The assumption is made to simplify the discussion, and it seems reasonable if the subarea is small enough such that highly attractive locations such as shopping malls do not appear as a clustered point in the subarea. However, other distributions may be applied but they are not studied here.

Given the relation of the network traffic and the spatial Poisson process, we can express mobile concentration in an subarea as a spatial Poisson process and estimate, in real time, its rate function based on the statistics of connection requests. In addition, the time dynamics of the rate function in each subarea can be expressed as a function of mobiles' mobility pattern, and which will be given in more detail in the next subsection. The network model based on the spatial Poisson process is formulated and given below.

Model Definition Let $i=1,2, \ldots, M$ indexes subareas, where $M$ is the number of subareas in the network, and $k=0,1, \ldots, 23$ denotes hours of a day, $\Pi_{k}^{i}$ is a spatial Poisson process with a constant rate $X_{k}^{i}$ in the subarea $i$ during the time interval $[k, k+1) . X_{k}=\left[X_{k}^{1}, X_{k}^{2}, \ldots, X_{k}^{M}\right]$ is a discrete time discrete state stochastic process, and, with Theorem 2, its state controls the rate of connection arrivals observed in each subarea. If there is only one subarea, $X_{k}^{1}$ is a hidden 
Markov chain observed through a Poisson process. For $M$ subareas, the state of $X_{k}^{i}$ for all subarea $i$ is modeled with the Influence model [9]. Suppose the subarea of interest is $i$, let $D(i)$ denotes the dependency of $i$, which is $i$ itself and its adjacent subareas, the transition probability for $X_{k}^{i}$ is

$$
P\left(X_{k+1}^{i} \mid X_{k}^{1}, \ldots, X_{k}^{M}\right)=\sum_{j \in D(i)} d_{i j} P\left(X_{k+1}^{i} \mid X_{k}^{j}\right),
$$

where $d_{i j}\left(\sum_{j} d_{i j}=1\right.$ and $\left.d_{i j}>0\right)$ and $P\left(X_{k+1}^{i} \mid X_{k}^{j}\right)$ are model parameters that are assumed known. (Some parameter estimation techniques can be found in [16].) Furthermore, the initial probability distribution $P\left(X_{k=0}^{i}\right)$ is also assumed known for all $i$.

\section{Justification of Influence Model and Model Reduction with Geographical Interpretation}

The premise of the mobility model are the mapping of the aggregate inter-subarea mobile movement to the networktraffic pattern, and the use of Markovian framework to model the aggregate movement. The implicit model assumptions made in (2) are the division of network into subareas, and the pattern of spatial interaction embedded in the parameters $d_{i j}$ and $P\left(X_{k+1}^{i} \mid X_{k}^{j}\right)$. In this subsection, we will provide justification of the assumptions that is in line with the Markovian framework applied in the geographical analysis such as the study of migration processes and commuting patterns, and discuss how model parameters could be reduced based on their geographical interpretation.

The use of Markovian nodal structure in geographical analysis is common and some examples can be found in [17], [18]. The most straightforward approach to characterize the network's nodal structure is via the use of transition matrix, i.e., $P\left(X_{k+1}^{1}, \ldots, X_{k+1}^{M} \mid X_{k}^{1}, \ldots, X_{k}^{M}\right)$, which contains the joint state dynamics of the network. However, such complete specification is not desirable because not only does the model dimension grow exponentially as the number of subareas, the interpretation of the model parameters is difficult. In Asavathiratham and et al [9], the Influence Model is introduced to describe interactions between many Markov chains. The model simplifies $P\left(X_{k+1}^{1}, \ldots, X_{k+1}^{M} \mid X_{k}^{1}, \ldots, X_{k}^{M}\right)$ to $M P\left(X_{k+1}^{i} \mid\right.$ $\left.X_{k}^{1}, \ldots, X_{k}^{M}\right)$, and for each $i, P\left(X_{k+1}^{i} \mid X_{k}^{1}, \ldots, X_{k}^{M}\right)$ is a convex combinations of $P\left(X_{k+1}^{i} \mid X_{k}^{j}\right)$ as shown in Eq. (2). Note that Eq. (2) degenerates to a standard Markov chain if $d_{i j}=1$ for $i=j$, and 0 otherwise.

In order to explain the spatial interaction among different subareas, we concern with attributal and associational properties of nodes [19]. Attributal properties refer to nodal characteristics due to the nodes itself (e.g. population), and associational properties refer to nodal characteristics due to the relationship between the nodes (e.g. distance). The associational properties is captured by the model parameter $d_{i j}$ and the attributal properties by the conditional probability $P\left(X_{k+1}^{i} \mid X_{k}^{j}\right)$.

The spatial interaction interpretation of the parameters is established by some geographical indices. In terms of mobility, for example, attributes such as residential or business district may be assigned to each node, and indices such as the number of bus routes may be assigned to each edge. Other choices such as the number of office buildings, residences, or the number of registered companies may also be used. According to their geographical interpretation, the model parameters can either be empirically estimated based on some geographical indices or be aggregated according to their attributes to reduce the model complexity.

The parameter $d_{i j}$ is constant factor indicating how often subarea $i$ is influenced by subarea $j$, and it can be interpreted as the probability of mobiles commuting from $j$ to $i$ as a function of routes connecting them. The parameter could be empirically estimated, for example, by counting the outgoing bus routes from one subarea to another. Consider a HomeWork mobility pattern seen in many mobility papers [20], [21] and a simple network consists of only three fully connected subareas, A, B, and C. Let $m_{A}, m_{B}$ and $m_{C}$ be the proportion of working people in $\mathrm{A}, \mathrm{B}$ and $\mathrm{C}$ respectively, and let $b_{i j}$ be the number of bus routes running from $j$ to $i$. The influence matrix can be constructed as

$$
\left(\begin{array}{ccc}
1-m_{A} & m_{A}\left(\frac{b_{B A}}{b_{B A}+b_{C A}}\right) & m_{A}\left(\frac{b_{C A}}{b_{B A}+b_{C A}}\right) \\
m_{B}\left(\frac{b_{A B}}{b_{A B}+b_{C B}}\right) & 1-m_{B} & m_{B}\left(\frac{b_{C B}}{b_{A B}+b_{C B}}\right) \\
m_{C}\left(\frac{b_{A C}}{b_{A C}+b_{B C}}\right) & m_{C}\left(\frac{b_{B C}}{b_{A C}+b_{B C}}\right) & 1-m_{C}
\end{array}\right) .
$$

On the other hand, the conditional probability $P\left(X_{k+1}^{i} \mid X_{k}^{j}\right)$ specifies the effects of the subarea $i$ on $j$, and it could be interpreted as the probability of mobiles switching between active and non-active talking states given that they're, for example, commuting from a business district to a residential area. In the same Home-Work setting, the complexity of the parameters can be reduced by assigning residential or business attribute to each subarea. The attribute is useful because, combining with the state of the subarea (mobile concentration), the time of the day could be inferred and thus the time variance in the parameter removed. Intuitively, residential area has high traffic, for example, in the morning and the evening when people are not working. Moreover, the attributes allow the application of the previous technique to empirically estimate the parameters.

In addition to the geographical interpretation that the Influence model posses and some ways to estimate and reduce the model parameters, the model complexity of the Influence model is another advantage in justifying its use. Suppose there are $M$ subareas and each subarea has $P$ states, the total number of model parameters are $M P^{2}+M^{2}$, which is greatly reduced from $P^{2 M}$ as in the case of complete specification.

\section{CDMA Network Assumptions and Outage Probability Cal- culation}

In this subsection, the models used for the CDMA network and the propagation are introduced, and the outage probability expression is derived. For adaptive sectoring, because the performance analysis concerns time scale in hours, many important CDMA physical layer effects, such as the signature sequence structure and the fast fading losses, are not included. The performance of the adaptive sectoring is studied with perfect power control, soft handoff and log-normal shadowing.

Propagation Model and Interference Calculation The propagation loss in general is modeled as the product of $\gamma$ th power 
of distance and a log-normal shadowing component [10]. Let $B_{n}$ denotes the location of base station $n$ and suppose an arbitrary mobile at location $z$, the following propagation loss model is assumed:

$\Gamma_{z}\left[B_{n}\right] \equiv d\left[z, B_{n}\right]^{\gamma} 10^{\xi / 10}=d\left[z, B_{n}\right]^{\gamma} 10^{a\left(\xi_{z} / 10\right)} 10^{b\left(\xi_{z, B_{n}} / 10\right)}$,

where $d\left[z, B_{n}\right]$ is the distance between $z$ and $B_{n}$, and $\gamma$ is the path loss exponent. The shadowing $\xi=a \xi_{z}+b \xi_{z, B_{n}}$ is the superposition of two components: $\xi_{z}$ is the shadowing in the near field of the mobile at point $z$, and $\xi_{z, B_{n}}$ is the shadowing in the wireless link between the mobile at $z$ and the base station $B_{n} . \xi_{z}$ and $\xi_{z, B_{n}}$ are independent Gaussian random variables with the following properties: $\mathbf{E}\left(\xi_{z}\right)=\mathbf{E}\left(\xi_{z, B_{n}}\right)=$ $0, \operatorname{Var}\left(\xi_{z}\right)=\operatorname{Var}\left(\xi_{z, B_{n}}\right)=\sigma^{2}$ and $\mathbf{E}\left(\xi_{z} \xi_{z, B_{n}}\right)=0$ for all $n$, and $\mathbf{E}\left(\xi_{z, B_{n}} \xi_{z, B_{m}}\right)=0$ for all $n \neq m$. In addition, $\xi_{z}$ and $\xi_{z, B_{n}}$ are assumed to have equal standard deviation, and thus $a^{2}=b^{2}=1 / 2$ is assumed.

With the propagation model in place, the base station that does the power control and the set of base stations that participate in soft handoff can be defined. Let $B=$ $\left[\begin{array}{lllll}B_{1} & B_{2} & \ldots & B_{N}\end{array}\right]$ denotes the $N$ base stations in the network, the path loss of a mobile at location $z$ to each of the base station is then $\Gamma_{z}[B]=\left[\Gamma_{z}\left[B_{1}\right] \Gamma_{z}\left[B_{2}\right] \ldots \Gamma_{z}\left[B_{N}\right]\right]$. The base station that power controls the mobile at $z$ is defined as $C_{z}=\arg \min _{i} \Gamma_{z}\left[B_{i}\right]$. As regard to soft handoff, assume $N_{s}$ base stations are involved, the soft handoff set, $\zeta_{z}$, is defined with respect to location $z$ as the set of $N_{s}$ base stations with the least path loss values in $\Gamma_{z}[B] . N_{s}$ is taken to be 2 in the rest of the paper.

Furthermore, the received power from the mobile at $z$ is power controlled to have magnitude of 1 at the base station $C_{z}$. As a result, a mobile at $z$ and power controlled by $C_{z}$ has to transmit with power $\Gamma_{z}\left[C_{z}\right]$, and the interference it induces on base station $B_{n}$ is equal to

$$
I= \begin{cases}\Gamma_{z}\left[C_{z}\right] / \Gamma_{z}\left[B_{n}\right], & \text { if } B_{n} \neq C_{z} \\ 1, & \text { if } B_{n}=C_{z}\end{cases}
$$

Interference Calculation Revisited with Spatial Poisson In Sec. II-B, Theorem 2 establishes that the mobile distribution in the network is spatial Poisson. The problem to be addressed is to revisit the interference calculation and take spatial Poisson into account. Fig. 2 shows the network model conceptually. Each point $z \in \Re^{2}$ is assigned a soft handoff set $\zeta_{z}$, which is represented as a diamond-shaped area; for any mobile within the diamond, the mobile is in soft handoff with the two base stations at the vertices, and power controlled by the one with smaller pathloss.

Suppose the interference at $B_{1}$ is of interest, which is the central base station in Fig. 2. Let $S$ be the set of all subareas in the shaded area $\mathrm{A}$, and $\Pi_{k}^{i}$ the subarea $i$ 's spatial Poisson process with rate $X_{k}^{i}$, the entire set of mobiles that is loading the sector is denoted as $\Pi_{k}=\bigcup_{i \in S} \Pi_{k}^{i}$. With (4), the total interference at the sector of $B_{1}$ is

$$
I\left[B_{1}\right]=\sum_{z \in \Pi_{k}} \Gamma_{z}\left[C_{z}\right] / \Gamma_{z}\left[B_{1}\right]
$$

According to [22], (5) can be classified into two components by identifying the two point patterns in $\Pi_{k}$; any point in $\Pi_{k}$

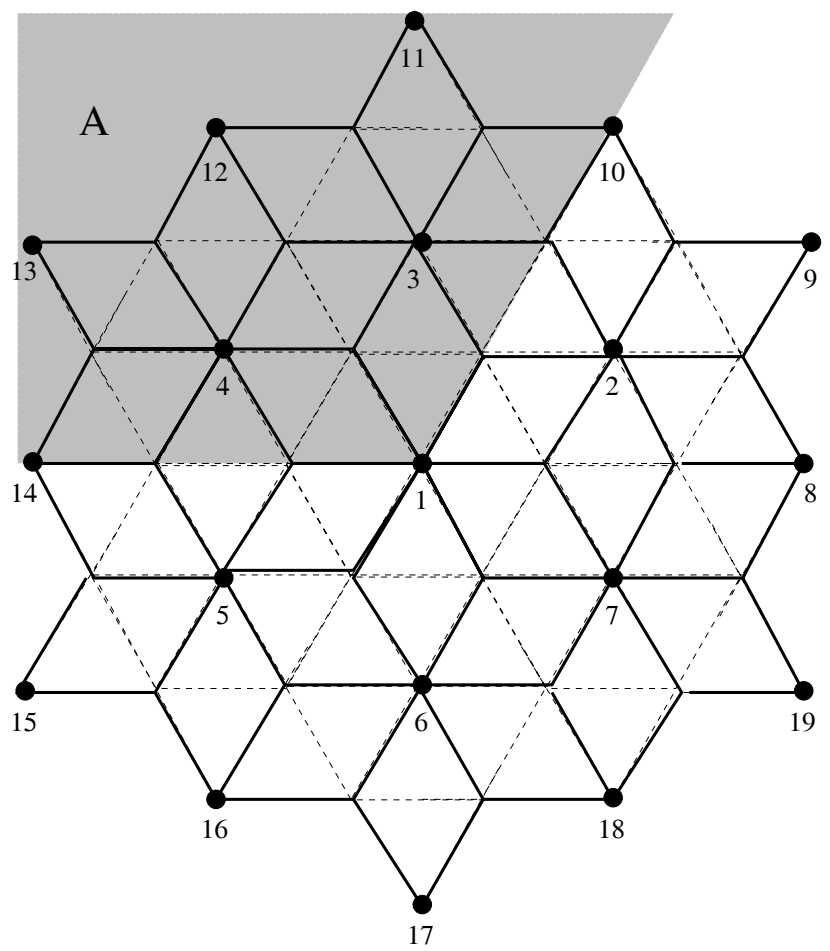

Fig. 2. Computation of the outage probability at the central base station 1 for a particular sector configuration covering the network area $A$. The diamonds shown in the figure represent the soft handoff set (Soft handoff is assumed to involve only two base stations in this paper), that is, for each mobile located in a diamond, the mobile is in soft handoff with the two base stations at the diamond's vertices, and power controlled by the one with smaller pathloss.

can either belong to a point pattern corresponding to $C_{z}=B_{1}$ $\left(\Pi_{k}\left[B_{1}\right]\right)$ or the one corresponding to $C_{z} \neq B_{1}\left(\Pi_{k}\left[\bar{B}_{1}\right]\right)$. The classification is achieved by the Poisson Marking theorem. According to the network definitions given above, the probability that a mobile at $z$ is power controlled by $B_{1}$ is $P\left(C_{z}=B_{1}\right)=P\left(\Gamma_{z}\left[B_{1}\right]<\Gamma_{z}\left[B_{n}\right]\right)$ and the probability of not power controlled by $B_{1}$ is $P\left(C_{z} \neq B_{1}\right)=P\left(\Gamma_{z}\left[B_{1}\right]>\right.$ $\left.\Gamma_{z}\left[B_{n}\right]\right)$ for all $n \in \zeta_{z}$ and $n \neq 1$. Furthermore, let $z \in \Re^{2}$ and define the mean measure of $\Pi_{k}$ as shown in (6), the two points patterns of mobiles in $\Pi_{k}$ are then classified as:

In-cell mobile is the point pattern $\Pi_{k}\left[B_{1}\right]$ with mean measure $m\left[B_{1}\right](d z)$ defined by

$d\left(m\left[B_{1}\right]\right)(z)=P\left(C_{z}=B_{1}\right) d m(z)$.

Other-cell mobile is the point pattern $\Pi_{k}\left[\bar{B}_{1}\right] \equiv \Pi_{k}-\Pi_{k}\left[B_{1}\right]$ with mean measure $m\left[\bar{B}_{1}\right](d z)$ defined by $d\left(m\left[\bar{B}_{1}\right]\right)(z)=$ $P\left(C_{z} \neq B_{1}\right) d m(z)=\left(1-P\left(C_{z}=B_{1}\right)\right) d m(z)$.

As a result, let $I^{i}\left[B_{1}\right]$ and $I^{o}\left[B_{1}\right]$ denote the in-cell and other-cell interference at $B_{1}$ respectively. The total interference at $B_{1}$ expressed in (5) is the summation of $I^{i}\left[B_{1}\right]$ and $I^{o}\left[B_{1}\right]$ :

$I\left[B_{1}\right]=I^{i}\left[B_{1}\right]+I^{o}\left[B_{1}\right]=\sum_{z \in \Pi_{k}\left[B_{1}\right]} 1+\sum_{z \in \Pi_{k}\left[\bar{B}_{1}\right]} \Gamma_{z}\left[C_{z}\right] / \Gamma_{z}\left[B_{1}\right]$.

The outage probability of the sector is then

$$
P\left(\sum_{z \in \Pi_{k}\left[B_{1}\right]} 1+\sum_{z \in \Pi_{k}\left[\bar{B}_{1}\right]} \Gamma_{z}\left[C_{z}\right] / \Gamma_{z}\left[B_{1}\right]>\alpha\right),
$$

where $\alpha$ is the threshold value for the total interference. The 


$$
m(d z)=\sum_{i \in S} X_{k}^{i} \delta(i, d z), \text { where } \delta(i, d z)= \begin{cases}1, & \text { if } d z \text { is in subarea } i \\ 0, & \text { otherwise }\end{cases}
$$

evaluation of (8) is presented in Sec. III-C. Note that the outage probability expression derived in this subsection is for a particular sector partition, however, it should be clear that the expression is identical for different partitions except the spatial Poisson process $\Pi_{k}$.

\section{AdAptive SEctoring Algorithm}

In the previous section, the adaptive sectoring problem is mapped to a shortest path problem of an acyclic network with changing weight matrix. In this section, the algorithms that work behind each model component is described. Sec. III-A provides an overview, Sec. III-B describes the MAP estimator of the spatial Poisson's rate function, Sec. III-C computes the outage probability and Sec. III-D discusses a possible antenna architecture for deploying the system discussed.

\section{A. Overview of the Adaptive Sectoring Algorithm in Pseu- docode}

Recall in Sec. II-B, the time $t$ of a day is divided into hourly intervals $k=\{0,1, \ldots, 23\}$. Let $M$ be the number of subareas and $N$ the number of sectors in a cell, the pseudocode of the adaptive sectoring algorithm is provided in Algorithm 1.

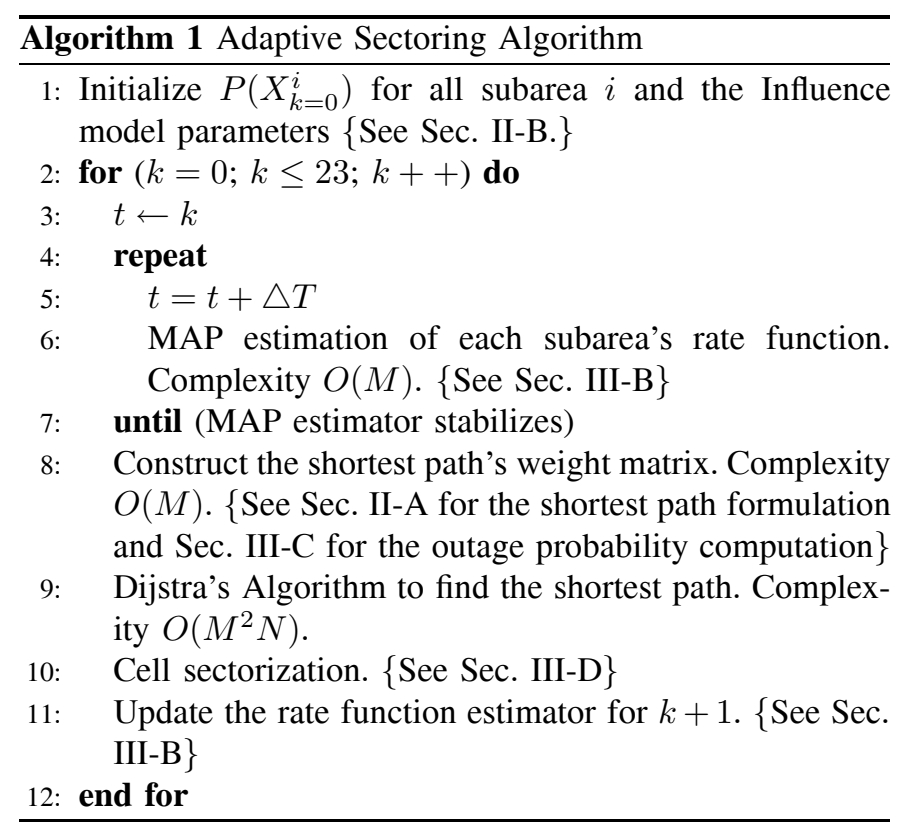

\section{B. MAP Estimator of Spatial Poisson's Rate Function}

In this subsection, a MAP estimator of $X_{k}^{i}$ given the traffic statistics is introduced. It should be noted that due to computation complexity, the joint a posteriori distribution of $X_{k}$ is not tracked, but only the a posteriori of $X_{k}^{i}$ (See Appendix A for details). Since the estimators for all subareas are equivalent, and for notational convenience, the subarea to be estimated is labeled as $X_{k}^{1}$, and the neighbors of $X_{k}^{1}$ are labeled as $X_{k}^{2}, X_{k}^{3}$ and $X_{k}^{4}$. The setup is illustrated in Fig. 7.
MAP Estimator Algorithm Let $\Pi_{k}^{i}$ be a spatial Poisson process with rate $X_{k}^{i}$ at the subarea $i$, and let $\left\{N_{k}^{i}(\sigma) ; k \leq \sigma<t\right\}$ denotes the observed path of $\Pi_{k}^{i}$ in the time interval $[k, t)$, i.e., the connection requests processed at the base station. The approximated a posteriori probability mass function of $X_{k}^{1}$ is iteratively calculated by the following algorithm.

For $t \in[k, k+1)$, define $\triangle N_{t}^{i}=N_{k}^{i}(t+\triangle t)-N_{k}^{i}(t)$, where $\triangle t$ is an arbitrary time interval, the a posteriori probability mass function $P\left(X_{k}^{i} \mid N_{k}^{i}(\sigma) ; k \leq \sigma<t\right)$, for $i=1,2,3,4$ is shown in (9), where $X_{k}^{i}=\mathbf{E}\left\{X_{k}^{i} \mid N_{k}^{i}(\sigma) ; k \leq \sigma<t\right\}$, and for $\triangle t$ small enough, $\triangle N_{t}^{i}$ is either 0 or 1 depending on occurrence or nonoccurrence of events. At the end of the time interval $[k, k+1)$, label $n_{1}=\left\{N_{k}^{1}(\sigma) ; k \leq \sigma<k+1\right\}$, $\ldots$, and $n_{4}=\left\{N_{k}^{4}(\sigma) ; k \leq \sigma<k+1\right\}$, the probability mass function of the subarea 1 at the beginning of the next time interval $[k+1, k+2)$ is

$$
\begin{aligned}
& P\left(X_{k+1}^{1}=x \mid n_{1}, n_{2}, n_{3}, n_{4}\right) \\
& \quad=\sum_{j=1}^{4} d_{1 j} \sum_{X_{k}^{j}} P\left(X_{k+1}^{1}=x \mid X_{k}^{j}\right) P\left(X_{k}^{j} \mid n_{j}\right),
\end{aligned}
$$

where $d_{1 j}$ and $P\left(X_{k+1}^{1} \mid X_{k}^{j}\right)$ are Influence model parameters. For $t \in[k+1, k+2)$, (9) again continuously update the a posteriori probability upon receiving connection requests. As a result, assuming the initial probability $P\left(X_{k=0}^{i}\right)$ is known for all $i$, the a posteriori probability of $X_{k}^{i}$ can be tracked for any time $t$, and thus the MAP estimator at time $t$ is

$$
\arg \max _{x} P\left(X_{k}^{1}=x \mid N_{k}^{1}(\sigma), \ldots, N_{k}^{4}(\sigma) ; k \leq \sigma<t\right)
$$

\section{Outage Probability Evaluation for Adaptive Sectoring}

In this subsection, the aim is to evaluate the outage probability (8) of an arbitrary sector configuration. The spatial Poisson distribution is assumed known, and its resulting outage probability is computed. Let $\Pi_{k}$ be the union of spatial Poisson processes loading a sector, the total interference received at the sector of base station $B_{1}$ is computed with (7) and the outage probability is

$$
\begin{aligned}
& P\left(I^{i}\left[B_{1}\right]+I^{o}\left[B_{1}\right]>\alpha\right) \\
& \quad=P\left(\sum_{z \in \Pi_{k}\left[B_{1}\right]} 1+\sum_{z \in \Pi_{k}\left[\bar{B}_{1}\right]} \Gamma_{z}\left[C_{z}\right] / \Gamma_{z}\left[B_{1}\right]>\alpha\right) .
\end{aligned}
$$

Recall $\Pi_{k}\left[B_{1}\right]$ is the mobile point pattern power controlled by $B_{1}$ and $\Pi_{k}\left[\bar{B}_{1}\right]$ is the point pattern not power controlled by $B_{1}$. It is obvious that the first term, $I^{i}\left[B_{1}\right]$, is a Poisson random variable. In addition, in order for the base station $B_{1}$ to be well defined, a condition that $I^{i}\left[B_{1}\right]>0$ should be imposed. Combining the two observations, the outage probability becomes

$$
\begin{aligned}
& P\left(I^{i}\left[B_{1}\right]+I^{o}\left[B_{1}\right]>\alpha \mid I^{i}\left[B_{1}\right]>0\right) \\
& \quad=\frac{e^{-u}}{1-e^{-u}} \sum_{j=1}^{\infty} \frac{(u)^{j}}{j !} P\left(I^{o}\left[B_{1}\right]>\alpha-(j-1)\right)
\end{aligned}
$$




$$
\begin{aligned}
& P\left(X_{k}^{i} \mid N_{k}^{i}(\sigma) ; k \leq \sigma<t+\triangle t\right) \\
& =P\left(X_{k}^{i} \mid N_{k}^{i}(\sigma) ; k \leq \sigma<t\right)\left\{1+\left(X_{k}^{i}-\bar{X}_{k}^{i}\right) \bar{X}_{k}^{i}{ }^{-1}\left(\triangle N_{t}^{i}-\bar{X}_{k}^{i} \triangle t\right)\right\}+o(\triangle t),
\end{aligned}
$$

where $u \equiv \mathbf{E}\left(I^{i}\left[B_{1}\right]\right)$. The exact expression for $P\left(I^{o}\left[B_{1}\right]\right)$ is difficult, however, from [12], [22], it is shown that Gaussian approximation can be applied; the approximation is motivated by the central limit theorem and it is treated rigorously in [12]. The mean and variance of the Gaussian approximation are the first and second cumulants of $I^{o}\left[B_{1}\right]$ respectively, and whose computation is shown in Theorem 3 and 4 .

Theorem 3 Let $\Pi_{k}\left[\bar{B}_{1}\right]$ be a Poisson point pattern on the network area A with mean measure $m\left[\bar{B}_{1}\right]$ and let $I^{o}\left[B_{1}\right]=\sum_{z \in \Pi_{k}\left[\bar{B}_{1}\right]} \Gamma_{z}\left[C_{z}\right] / \Gamma_{z}\left[B_{1}\right]$. If $\int_{A} \min \left(\left|\Gamma_{z}\left[C_{z}\right] / \Gamma_{z}\left[B_{1}\right]\right|, 1\right) m\left[\bar{B}_{1}\right](d z)<\infty$ holds, then for any complex number $s$,

$$
\begin{aligned}
& E\left(\exp \left(s I^{o}\left[B_{1}\right]\right)\right) \\
& \quad=\exp \left(\int_{A}\left[\exp \left(s \Gamma_{z}\left[C_{z}\right] / \Gamma_{z}\left[B_{1}\right]\right)-1\right] m\left[\overline{B_{1}}\right](d z)\right) .
\end{aligned}
$$

Proof The proof can be found in [22].

The hypothesis holds since the area A is finite (only the first layer of interference is considered in this paper), and the mean measure of the spatial Poisson process has finite states.

Theorem 4 Divide the network area $A$ into $A_{\text {in-cell }}$ and $A_{\text {other-cell}}$, where in-cell (other-cell) is defined by the inclusion (exclusion) of $B_{1}$ in the soft handoff set $\zeta_{z}$ at the location $z$. Label the soft handoff base stations in $A_{\text {in-cell }}$ as $C_{z}$ and $B_{1}$, and the base stations in $A_{\text {other-cell }}$ as $B_{m}$ and $B_{n}$ (Assume soft handoff of 2 base stations), let $\kappa_{c}$ denotes the $c^{t h}$ cumulant of $I^{o}\left[B_{1}\right]$, and $\phi_{z}\left[B_{1}\right]=\frac{\Gamma_{z}\left[C_{z}\right]}{\Gamma_{z}\left[B_{1}\right]}$ if $C_{z} \neq$ $B_{1}$ and 0 otherwise, yielding (12), where $m(d z)$ is defined in (6), $\beta \equiv \ln 10 / 10$ and $M_{B_{l}} \equiv 10 \gamma \log _{10} d\left[z, B_{l}\right]$.

Proof The proof can be found in [22].

With (12), $I^{o}\left[B_{1}\right]$ 's mean and variance, $\kappa_{1}$ and $\kappa_{2}$ respectively, are calculated, and the outage probability becomes:

$P\left(I^{i}\left[B_{1}\right]+I^{o}\left[B_{1}\right]>\alpha \mid I^{i}\left[B_{1}\right]>0\right)=\frac{e^{-m}}{1-e^{-m}} \sum_{j=1}^{\infty} \frac{m^{j}}{j !} Q\left(\tilde{y}_{j}\right)$

where $\tilde{y_{j}} \equiv\left(\alpha-j+1-\kappa_{1}\right) / \sqrt{\kappa_{2}}, m$ is the mean of $\Pi_{k}\left[B_{1}\right]$ and $Q$ is the Q-function for the standard normal distribution.

From the above equations, it can be seen that if the rate function of the spatial Poisson process is known, the cost function for each sectoring configuration can be computed. However, the computation requires two numerical integration: one for the in-cell mobiles and the other for the other-cell mobiles. The numerical integration process is computationally intensive and time consuming. Fortunately, because the rate function of the spatial Poisson process is assumed to be constant over each subarea, the integration can be precomputed, and real time operation has computational complexity linear to the number of subareas in $\Pi_{k}$.

\section{Antenna Architecture}

The antenna architecture that support the adaptive sectoring algorithm can be considered as a migration from a fixed 3-sector CDMA system to a switched-beam smart antenna system. A switched-beam system has a set of predefined antenna patterns, and it serves each mobile with the dynamically chosen antenna pattern of best signal. However, in many cases, such individual-based adaptation is not necessary. Therefore, as an intermediate stage between the fixed sectoring and the switched beam antenna system, adaptive sectoring forms sectors by combining subset of the switch-beams. The implementation can be build on top of existing fixed sectoring system by deploying circular antenna array and a beam forming network per sector [23]. Each sector is identified by its pilot signal and softer handoff is used when mobiles travel between sectors. In addition, since the location of mobiles can be identified by the beam with the strongest signal strength, the statistics of each subarea's network traffic (Recall Sec. III-B) can be collected by summing up the connection requests in the beams making up the subarea.

\section{Simulation Results}

In this section, the focus is on the numerical studies of the adaptive sectoring algorithm. The analysis consists of two parts: Sec. IV-A simulates the traffic tracking with the mobility-enhanced traffic model as described in Sec. III-B, and Sec. IV-B simulates a hot-spot scenario where a comparison in performance of adaptive and fixed sectoring is made.

\section{A. Simulation of Spatial Poisson Estimation}

As described in Sec. II-B, the network traffic is a spatial Poisson process with rate function modeled according to the Influence Model. Ideally, the parameters of the Influence model can be learned from the actual traffic statistics using particle filtering or EM algorithm [16]. However, in this paper, the model parameters are assumed known from empirical data, and a hypothetical network is used to simulate the network traffic.

The hypothetical network consists of 10 adjacent subareas, and the Influence model parameters are arbitrarily chosen. The rate function $X_{k}^{i}$ in each subarea is assumed to have three states $\{$ High, Medium, Low $\}$. Fig. 3 illustrates the tracking of two subareas for four time slices. The blue line is the true state, and the green dotted line is the MAP estimator. It can be seen that the estimator follows the true state nicely except the first few minutes after each time the rate function changes value (on a hourly basis). The reason is that the estimation equation is formulated in a finite difference form, and update is done only upon new arrival of connection requests. As a result, a certain convergence time is needed for the MAP estimator to reach the true value.

Fig. 4 illustrates the real time tracking with the finite difference equation (9) of the two subareas during the first time interval. The top (bottom) plot shows the convergence of the probability mass function to the state High (Low) as connection requests are accumulated. It can be observed that the 


$$
\begin{aligned}
\kappa_{c} & =\frac{d^{c}}{d s^{c}}\left(\left.\ln \mathbf{E}\left(\exp \left(s I^{o}\left[B_{1}\right]\right)\right)\right|_{s=0}\right. \\
& =\int_{A} \mathbf{E}\left(\phi_{z}\left[B_{1}\right]\right)^{c} m(d z)=\int_{A} \mathbf{E}\left(\left(\frac{\Gamma_{z}\left[C_{z}\right]}{\Gamma_{z}\left[B_{1}\right]}\right)^{c} ; \Gamma_{z}\left[C_{z}\right]<\Gamma_{z}\left[B_{1}\right]\right) m(d z) \\
& =\int_{A_{\text {in-cell }}} \mathbf{E}\left(\left(\frac{\Gamma_{z}\left[C_{z}\right]}{\Gamma_{z}\left[B_{1}\right]}\right)^{c} ; \Gamma_{z}\left[C_{z}\right]<\Gamma_{z}\left[B_{1}\right]\right) m(d z) \\
& +\int_{A_{\text {other-cell }}} \mathbf{E}\left(\left(\frac{\Gamma_{z}\left[B_{m}\right]}{\Gamma_{z}\left[B_{1}\right]}\right)^{c} ; \Gamma_{z}\left[B_{m}\right]<\Gamma_{z}\left[B_{n}\right]\right) m(d z) \\
& +\int_{A_{\text {other-cell }}} \mathbf{E}\left(\left(\frac{\Gamma_{z}\left[B_{n}\right]}{\Gamma_{z}\left[B_{1}\right]}\right)^{c} ; \Gamma_{z}\left[B_{n}\right]<\Gamma_{z}\left[B_{m}\right]\right) m(d z) \\
& =\exp \left((c \beta b \sigma)^{2}\right) \int_{A_{\text {in-cell }}}\left(\frac{d\left[z, C_{z}\right]}{d\left[z, B_{1}\right]}\right)^{c \gamma} Q\left(\sqrt{2} c \beta b \sigma+\frac{M_{C_{z}}-M_{B_{1}}}{\sqrt{2} \sigma b}\right) m(d z) \\
& +2 \exp \left((c \beta b \sigma)^{2}\right) \int_{A_{\text {other }- \text { cell }}}\left(\frac{d\left[z, B_{m}\right]}{d\left[z, B_{1}\right]}\right)^{c \gamma} Q\left(\frac{c \beta b \sigma}{\sqrt{2}}+\frac{M_{B_{m}}-M_{B_{n}}}{\sqrt{2} b \sigma}\right) m(d z),
\end{aligned}
$$
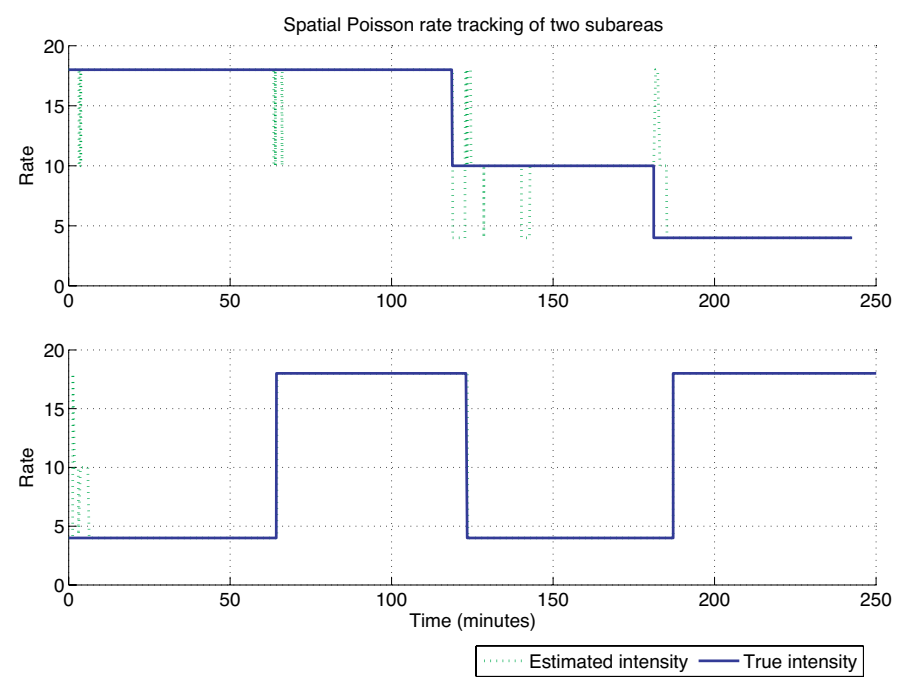

Fig. 3. The real time MAP estimations of the traffic in 2 subareas are plotted for four 60-minute periods. The realizations of the intensity functions in subareas are generated using the Influence model, and (11) is applied to follow. The solid line is the simulated realization, and the dotted line is the MAP estimator value.

convergence time is inversely proportional to the magnitude of the rate function. The tracking converges within 4 minutes when the subarea is in state High, while the tracking of the state Low takes approximately 40 minutes. However, since the MAP estimator depends only on the absolute difference between the state probabilities, the estimation yields accurate result as long as the true state has the highest probability.

\section{B. Simulation of Adaptive Sectorization}

The typical problem of nonuniform traffic is manifested in the generation of hot spots. In this subsection, the response of the adaptive sectoring algorithm is studied against a hot spot scenario, where a comparison in network capacity of the adaptive and the fixed sectoring is made. Fig. 2 illustrates the network model. The network consists of 19 cells and each cell has radius of one. The value of the path-loss exponent, $\gamma$, is assumed to be 4 , and the required SIR is set
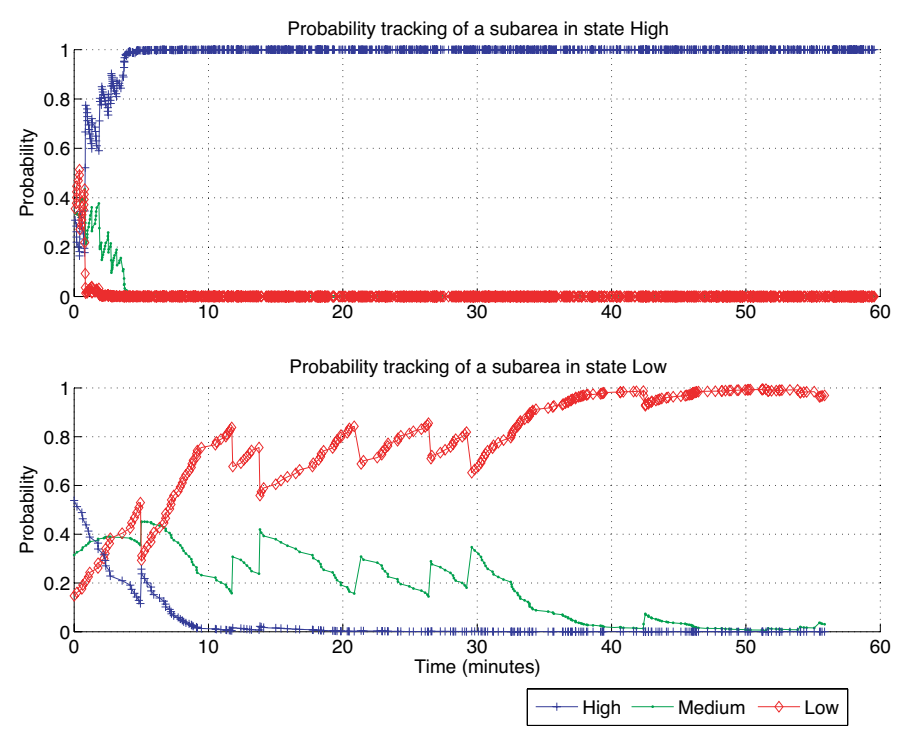

Fig. 4. The two plots illustrates the real time tracking of the a posteriori probability of the two subareas in Fig. 3 for the first 60-minute period. (9) is applied to update the a posteriori probability mass function as traffic data is accumulated. It is evident from the figure that the convergence time in tracking is inversely proportional to the rate function.

to $7 \mathrm{~dB} / 128$, which corresponds to a despread SIR of $7 \mathrm{~dB}$ when the spreading factor is 128 . Furthermore, the shadowing component in the propagation uncertainty is taken to have standard deviation of $8 \mathrm{~dB}$. Under the network assumptions made, the outage probability of different sector configurations under uniform traffic is illustrated in Fig. 5. It is obvious that the sector with angular span of 4 subareas has the steepest slope, and the sector with one subarea is the smoothest.

Suppose the cell of interest is the central cell, the hot spot scenario considered is to increase the rate function of its two neighboring cells, and determine how adaptive sectoring can mitigate the effect. Fig. 6 illustrates the difference in outage probabilities between the fixed and the adaptive sectoring. In the fixed sectoring case, when the rate function is gradually increased, the sector closest to the hot spot experiences high outage probability while the other two sectors have all the 


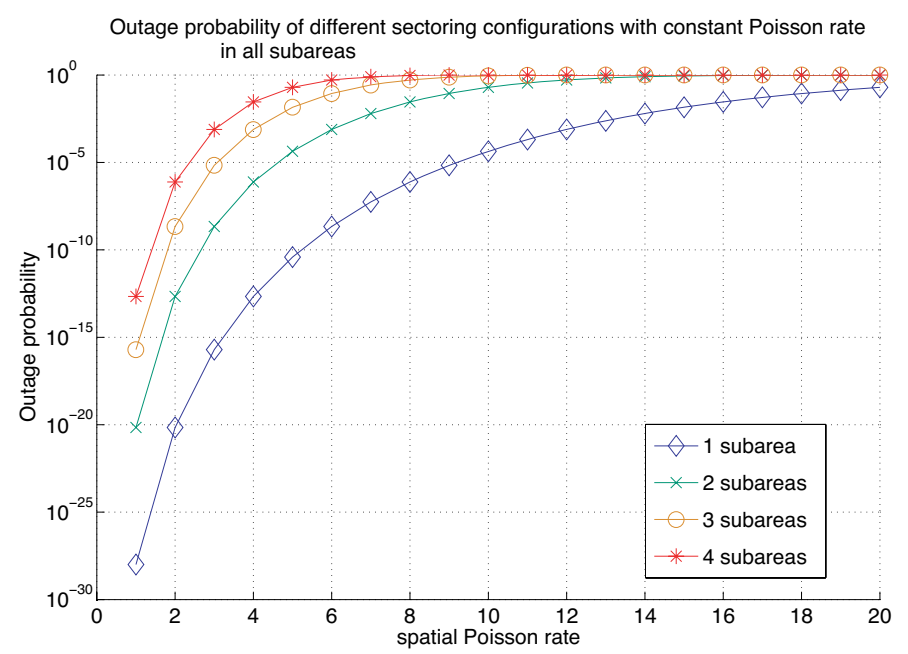

Fig. 5. Outage probability of sector configuration consisting of 1 to 4 subareas under constant spatial Poisson rate.
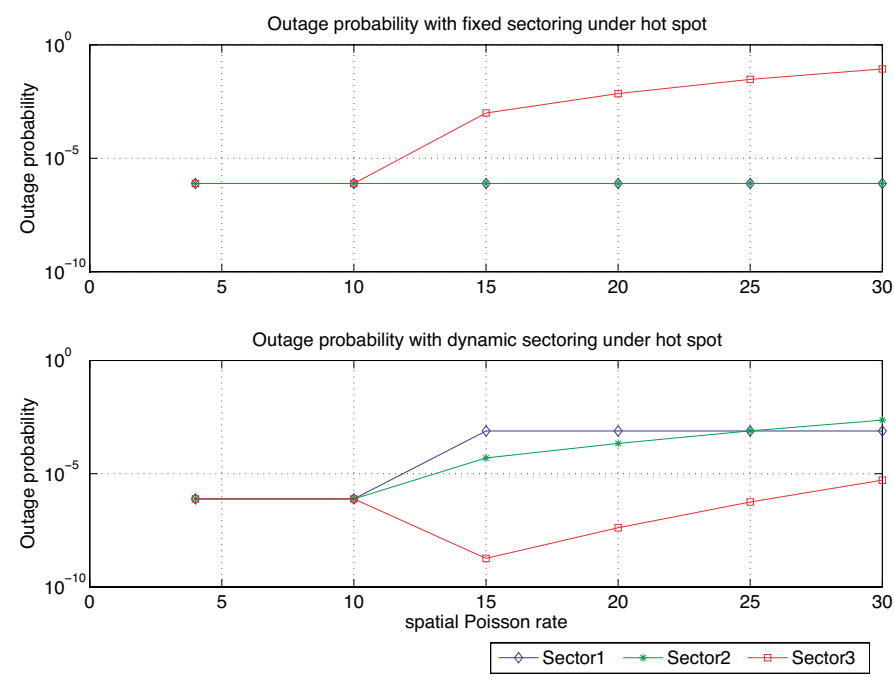

Fig. 6. Comparison of system performance with fixed and dynamic sectoring under hot spot condition. It can be observed that dynamic sectoring balances the traffic and keep the outage probabilities of the three sectors under $1 \%$, where the loaded sector in fixed sectoring has approximately $9 \%$ outage probability.

unutilized resources. On the other hand, the adaptive sectoring algorithm narrows the loaded sector when its outage probability starts to rise, and share the load among the three sectors. It is observed that even though the outage probabilities have risen in the other two sectors, they are well below $1 \%$; the outage probability in the fixed sectoring case has risen to approximately $9 \%$.

\section{CONCLUSION}

In this paper, the adaptive sectoring problem is formulated as a shortest path problem. The weight matrix of the acyclic network constructed depends on mobiles' spatial distribution, and which is estimated by a MAP estimator as a function of the network traffic. The real time tracking of the network traffic enables the system to minimize the outage probability at a base station by responding to non-stationary and non-uniform mobile distribution with adaptive sectoring.
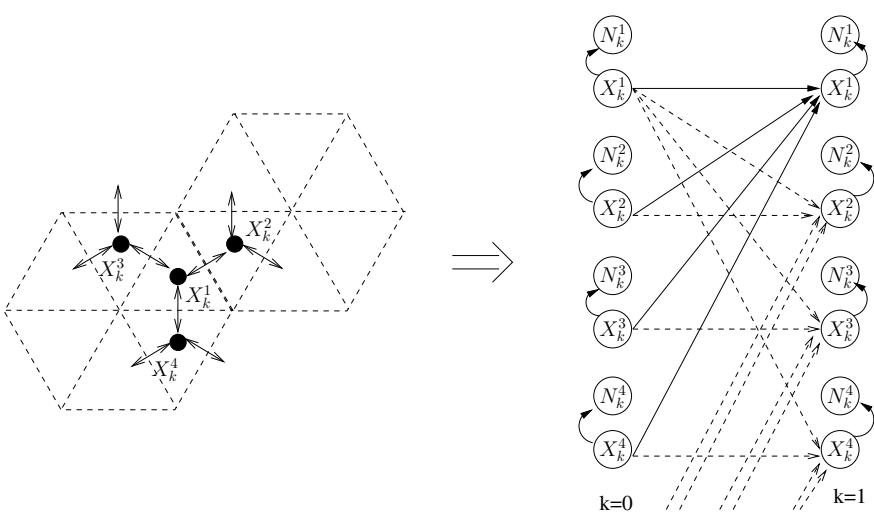

Fig. 7. Label the subarea of interest as $X_{k}^{1}$, the network on the left illustrates the dependency structure of $X_{k}^{1}$ on its neighbors. Only 4 subareas are shown because it is assumed that only adjacent subareas have influence on the dynamics of $X_{k}^{1}$ (Recall Sec. II-B). The dynamic Bayesian network on the right models the evolution of $X_{k}^{1}$. The solid lines indicate the dependency structure of $X_{k}^{1}$ on itself and its neighbors' previous states. The dotted lines indicate the dependency structure of other subareas.

The simulation on the tracking of the spatial Poisson process' rate functions has shown rapid convergence when the rate function is high. However, convergence is slow when the rate is low. Fortunately, slow convergence does not mean bad performance. Accurate estimation is made as long as the true state has the highest probability. Furthermore, the simulation of hot spot scenario has demonstrated the ability of the adaptive sectoring to cope with nonuniform traffic distribution. The adaptive sectoring balances the load between sectors such that no sector has outage probability exceeding $1 \%$, while the fixed sectoring scheme experiences outage of approximately $9 \%$.

Future work with the model developed is parameter estimation with real traffic data. Currently, the model parameters are assumed known, and each subarea is assumed to have the same state space. However, the Influence Model is very flexible. It is possible to have different state space for different subareas, and have the model parameters estimated based on real time traffic data.

\section{APPENDIX}

\section{A. Derivation of MAP Estimator}

In this Appendix, the MAP estimator introduced in Sec. III-B is derived. The estimator developed is similar to other HMM-type estimators except 1 ) The a posteriori probability is updated continuously in discrete steps during the time interval $[k, k+1)$ instead of once every $k$, and 2) The a posteriori probability of each individual subarea is tracked instead of the joint a posteriori probability of all the subareas. Tracking joint a posteriori probability distribution is too computationally intensive for real time applications.

For notational convenience, the state of the subarea of interest is labelled as $X_{k}^{1}$ and its neighbors as $X_{k}^{2}, X_{k}^{3}$ and $X_{k}^{4}$. The time evolution of $X_{k}^{1}$ and its dependency are illustrated in Fig. 7, where the solid lines show the dependency of $X_{k}^{1}$ at $k+1$ on itself and its neighbors' previous state. (The dotted lines refer to other subareas' dependency, and they are irrelevant in estimating $X_{k}^{1}$.) For each subarea $i, X_{k}^{i}$ is hidden, and only the 


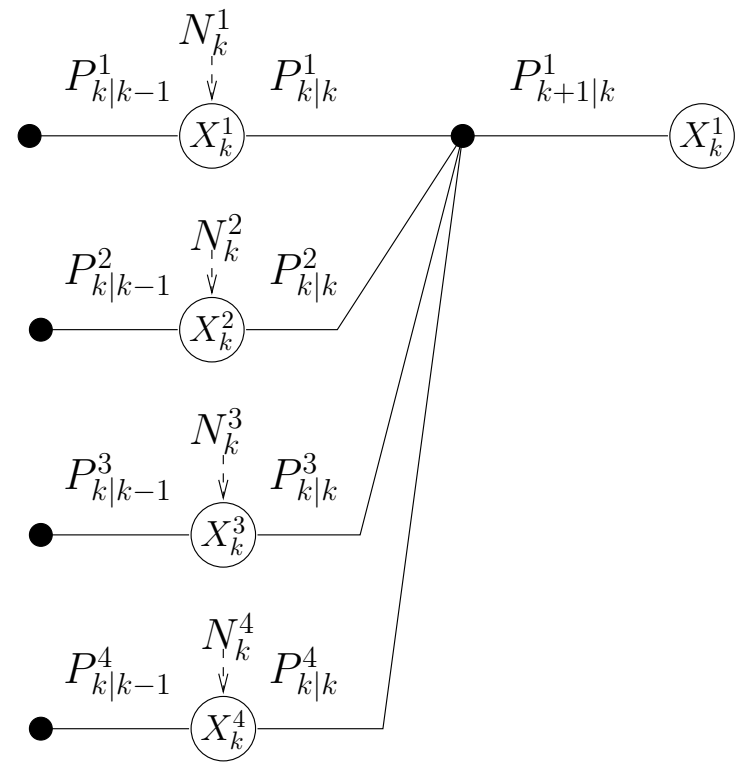

$\mathrm{k}=0 \quad \mathrm{k}=1$

Fig. 8. Factor graph representation of the Bayesian network in Fig. 7. The $X_{k}^{1}$ is isolated and the conditional probabilities relevant to the estimation of $X_{k}^{1}$ are explicitly illustrated.

connection request, $N_{k}^{i}$, is observed. Denote the a posteriori probability distribution of $X_{k}^{1}$ given all the network traffic up to time $k$ as $P_{k \mid k}^{1}=P\left(X_{k}^{1} \mid N_{0: k}^{1}, N_{0: k}^{2}, N_{0: k}^{3}, N_{0: k}^{4}\right)$, and the prediction as $P_{k+1 \mid k}=P\left(X_{k+1}^{1} \mid N_{0: k}^{1}, N_{0: k}^{2}, N_{0: k}^{3}, N_{0: k}^{4}\right)$, where $N_{0: k}^{i}=\left(N_{0}^{i} N_{1}^{i} \ldots N_{k}^{i}\right)$. The recursive computation of $P_{k \mid k}^{1}$ and $P_{k+1 \mid k}^{1}$ tracks the a posteriori probability for subarea 1. Fig. 8 illustrates the dynamics as a factor graph with the a posteriori distribution explicitly stated.

The prediction step can be computed easily given $P_{k \mid k}^{i}$ for $i=1,2,3,4$. Denote $\sum_{X_{k}} \equiv \sum_{X_{k}^{1}} \sum_{X_{k}^{2}} \cdots \sum_{X_{k}^{4}}, P_{k+1 \mid k}^{1}$ can be written as

$$
\begin{aligned}
P_{k+1 \mid k}^{1} & =\sum_{X_{k}} P\left(X_{k+1}^{1} \mid X_{k}^{1}, X_{k}^{2}, X_{k}^{3}, X_{k}^{4}\right) P_{k \mid k}^{1} P_{k \mid k}^{2} P_{k \mid k}^{3} P_{k \mid k}^{4} \\
& =\sum_{X_{k}}\left(\sum_{j} d_{1 j} P\left(X_{k+1}^{1} \mid X_{k}^{j}\right)\right) P_{k \mid k}^{1} P_{k \mid k}^{2} P_{k \mid k}^{3} P_{k \mid k}^{4} \\
& =\sum_{j} d_{1 j}\left(\sum_{X_{k}^{j}} P\left(X_{k+1}^{1} \mid X_{k}^{j}\right) P_{k \mid k}^{j}\right)
\end{aligned}
$$

where the second step uses the Influence model representation, and both $d_{1 j}$ and $P\left(X_{k+1}^{1} \mid X_{k}^{j}\right)$ are defined model parameters. The updating step, the computation of $P_{k \mid k}^{i}$ from the previous prediction $P_{k \mid k-1}^{i}$, is illustrated in the following Theorem.

Theorem Suppose $N_{k}^{i}(t)$ is doubly stochastic Poisson with rate $X_{k}^{i}$, and $X_{k}^{i}$ is a random variable. If we let $P_{t}\left(X_{k}^{i} \mid N_{k}^{i}(\sigma) ; k \leq \sigma<t\right)$ denote the conditional probability density function for $X_{k}^{i}$ given the connection request statistics $\left\{N_{k}^{i}(\sigma) ; k \leq \sigma<t\right\}$, then we obtain (14), with $P\left(X_{k}^{i} \mid N_{k}^{i}(\sigma) ; \sigma=k\right)=P_{k \mid k-1}^{i}$, and $\bar{X}_{k}^{i}=$ $E\left\{X_{k}^{i} \mid N_{k}^{i}(\sigma) ; k \leq \sigma<t\right\}$.

Proof The proof can be found in [24].

Eq. (14) can also be viewed as defining an updating algorithm according to which the prior density $P_{k \mid k-1}^{i}$ is propagated forward in time to form the a posterior density $P_{k \mid k}^{i}$ as data are accumulated. For this interpretation, it is convenient to rewrite (14) in the finite difference form shown in (15). For $\triangle t$ sufficiently small, the term $\mathrm{o}(\triangle t)$ can be disregarded and $\triangle N_{t}^{i}=N_{k}^{i}(t+\triangle t)-N_{k}^{i}(t)$ will be either zero or one according to the nonoccurrence or occurrence of a point in $[t, t+\triangle t)$. As $\sigma$ reaches time $k+1, P\left(X_{k}^{i} \mid N_{k}^{i}(\sigma)\right)=P_{k \mid k}^{i}$, which completes the cycle.

\section{REFERENCES}

[1] S. Dennett, "The CDMA2000 ITU-R RTT candidate submission (0.18)," TIA, Tech. Rep., July 1998.

[2] J. C. Liberti and T. S. Rappaport, Smart Antennas for Wireless Communications: IS-95 and Third Generation CDMA Applications. Prentice Hall PTR, 1999.

[3] 3GPP, "Technical specification group radio access network, physical channels and mapping of transport channels onto physical channels (FDD), TS 25.221 V3.2.0 (2000-03)."

[4] C. U. Saraydar and A. Yener, "Adaptive cell sectorization for cdma systems," IEEE J. Select. Areas Commun., vol. 19, pp. 1041-1051, June 2001.

[5] F. M. R. Giuliano and F. Vatalaro, "Smart cell sectorization for third generation cdma systems," Wireless Commun. and Mobile Comput., vol. 2, pp. 253-267, May 2002.

[6] A. Ahmad, "A CDMA network architecture using optimized sectoring," IEEE Trans. Veh. Technol., vol. 51, pp. 404-410, May 2002.

[7] J. C. Yun and et al., "Traffic balancing performance of adaptive sectorized systems," in Proc. Vehicular Technology Conference, vol. 3 , pp. 1878-1881, Sept. 2002.

[8] D. Tang and M. Baker, "Analysis of a metropolitan-area wireless network," Wireless Networks, vol. 8, pp. 107-120, Mar. 2002.

[9] C. Asavathiratham, S. Roy, B. Lesieutre, and G. Verghese, "The influence model," IEEE Control Syst. Mag., vol. 21, pp. 52-64, Dec. 2001.

[10] A. J. Viterbi, CDMA: Principles of Spread Spectrum Communication. Addison-Wesley Publishing Company, 1995.

[11] L. Korowajczuk and et al., Designing CDMA2000 Systems. John Wiley and Sons, Ltd, 2004.

[12] J. S. Evans and D. Everitt, "On the teletraffic of CDMA cellular networks," IEEE Trans. Veh. Technol., vol. 48, pp. 153-165, Jan. 1999.

[13] C. D. Simone and et al., "Fair dissections of spiders, worms, and caterpillars," Networks, vol. 20, pp. 323-344, 1990.

[14] J. F. C. Kingman, Poisson Processes. Clarendon Press, Oxford, 1993.

[15] M. Sexton and A. Reid, Broadband Networking. Artech House, Inc., 1997.

[16] S. Basu, T. Choudhury, B. Clarkson, and A. Pentland, "Learning human interactions with the influence model," MIT Media Laboratory, Tech. Rep., June 2001.

[17] C. Dennis, D. Marsland, and T. Cockett, "Central place practice: shopping centre attractiveness measures, hinterland boundaries and the UK retail hierarchy," J. Retailing and Consumer Services, vol. 9, pp. 185-199, 2002.

[18] G. Navdal, I. Thorsen, and J. Uboe, "Modeling spatial structures through equilibrium states for transitioin matrices," J. Regional Science, vol. 36, pp. 171-196, 1996

[19] L. A. Brown and F. E. Horton, "Functional distance: An operational approach," Geographical Analysis, vol. 2, pp. 76-83, 1970.

[20] D. Lam, D. Cox, and J. Widom, "Teletraffic modeling for personal communications services," IEEE Commun. Mag., vol. 35, pp. 79-87, Feb. 1997.

[21] A. Kumar, M. N. Umesh, and R. Jha, "Mobility modeling of rush hour traffic for location area design in cellular networks," in Proc. Proc. 3rd ACM International Workshop on Wireless Mobile Multimedia. New York: ACM Press, 2000, pp. 48-54.

[22] C. C. Chan and S. V. Hanly, "Calculating the outage probability in a cdma network with spatial poisson traffic," IEEE Trans. Veh. Technol., vol. 50, pp. 183-203, Jan. 2001.

[23] M. Mahmoudi, E. S. Sousa, and H. Alavi, "Adaptive sector size control in a cdma system using butler matrix," IEEE Trans. Veh. Technol., vol. 2, pp. 1355-1359, May 1999.

[24] D. L. Snyder and M. I. Miller, Random Point Processes in Time and Space. Springer-Verlag, 1991. 


$$
\begin{aligned}
& d p\left(X_{k}^{i} \mid N_{k}^{i}(\sigma) ; k \leq \sigma<t\right)=p\left(X_{k}^{i} \mid N_{k}^{i}(\sigma) ; k \leq \sigma<t\right)\left(X_{k}^{i}-\bar{X}_{k}^{i}\right) \bar{X}_{k}^{i}{ }^{-1}\left(d N_{t}^{i}-\bar{X}_{k}^{i} d t\right) \\
& \quad P\left(X_{k}^{i} \mid N_{k}^{i}(\sigma) ; k \leq \sigma<t+\triangle t\right) \\
& \quad=P\left(X_{k}^{i} \mid N_{k}^{i}(\sigma) ; k \leq \sigma<t\right)\left\{1+\left(X_{k}^{i}-\bar{X}_{k}^{i}\right) \bar{X}_{k}^{i}{ }^{-1}\left(\triangle N_{t}^{i}-\bar{X}_{k}^{i} \triangle t\right)\right\}+o(\triangle t)
\end{aligned}
$$

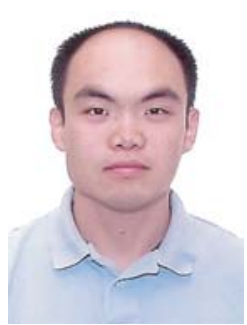

Alex Wang was born in 1979 in Taiwan. He received his bachelor degree (with honours) in Engineering Physics with Commerce Minor and his Master degree in Electrical and Computer Engineering from the University of British Columbia in 2003 and 2005 respectively. He is currently pursuing his Ph.D. degree in Statistical Signal Processing, under the supervision of Dr. Vikram Krishnamurthy, in the University of British Columbia. His research interests include radar signal processing, syntactic pattern recognition and uncertain reasoning.

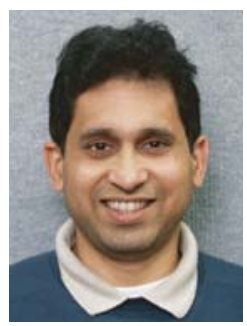

Vikram Krishnamurthy (S'90-M'91-SM'99-F'05) was born in 1966. He received his bachelor's degree from the University of Auckland, New Zealand in 1988, and Ph.D. from the Australian National University, Canberra, in 1992. Since 2002, he has been a professor and Canada Research Chair at the Department of Electrical Engineering, University of British Columbia, Vancouver, Canada. Prior to 2002, he was a chaired professor at the Department of Electrical and Electronic Engineering, University of Melbourne, Australia, where he also served as deputy head of department. His current research interests include stochastic dynamical systems for modeling of biological ion channels and biosensors, stochastic optimization and scheduling, and statistical signal processing. Dr. Krishnamurthy has served as associate editor for several journals including IEEE Transactions Automatic Control, IEEE Transactions on Signal Processing, IEEE Transactions Aerospace and Electronic Systems, IEEE Transactions Circuits and Systems B, IEEE Transactions Nanobioscience, and Systems and Control Letters. He is co-editor with S. H. Chung and O. Andersen of the book Biological Membrane Ion Channels - Dynamics Structure and Applications, published by Springer-Verlag in 2006. 